\title{
Antifungal properties of Aloe vera through in vitro and in vivo screening against postharvest pathogens of papaya fruit
}

\begin{abstract}
Postharvest fruit disease in papaya (Carica papayaL.) caused by fungus is one of the major problems in the fruit industry. The aim of the study is to determine the major postharvest fungal pathogen isolated from papaya fruit and to investigate the antifungal effects of Aloe vera on those pathogens. In this study, the fungi were isolated and identified through molecular identification as Fusariumsp., Lasiodiplodia theobromae, Aspergillus niger, and Colletotrichum gloeosporioides which are believed to responsible for postharvest decay in papaya fruits. Two types of Aloe vera extract (Aloe barbadensis Miller); fresh Aloe vera and food grade were used to evaluate their efficacy in inhibiting fungal growth thereby slowing down fruit decay. Both Aloe vera with different concentrations $(0,15 \%, 25 \%$, and $50 \%(\mathrm{v} / \mathrm{v}))$ were tested against mycelium growth of four pathogenic fungi of papaya fruit. Mycelial growth of fungi was inhibited by both fresh filtered and food grade Aloe vera amended with PDA media in a dose-response manner. Both fresh and food grade Aloe vera gel were able to prevent wound infection on papaya fruit inoculated with Fusariumsp., Aspergillus niger, Colletotrichum gloeosporioides, and Lasiodiplodia theobromae for $72 \mathrm{~h}$ after inoculation of the fungi. In summary, the study paves the way of Aloe vera as a potential edible coating for controlling the postharvest fungus of papaya fruit.
\end{abstract}

Keyword: Aloe vera; Postharvest; Papaya; Antifungal; Inhibition 